\title{
Catalytic asymmetric tandem Friedel-Crafts alkylation/Michael addition reaction for the synthesis of highly functionalized chromans
}

\author{
Jiahuan Peng and Da-Ming Du*
}

Open Access

\author{
Full Research Paper \\ Address: \\ School of Chemical Engineering and Environment, Beijing Institute of \\ Technology, Beijing 100081, China \\ Email: \\ Da-Ming Du* - dudm@bit.edu.cn \\ * Corresponding author \\ Keywords: \\ asymmetric catalysis; bis(oxazoline); chroman; Friedel-Crafts \\ alkylation; tandem reaction
}

\author{
Beilstein J. Org. Chem. 2013, 9, 1210-1216. \\ doi:10.3762/bjoc.9.137 \\ Received: 09 March 2013 \\ Accepted: 23 May 2013 \\ Published: 24 June 2013 \\ Associate Editor: J. N. Johnston \\ (c) 2013 Peng and Du; licensee Beilstein-Institut. \\ License and terms: see end of document.
}

\begin{abstract}
The enantioselective tandem Friedel-Crafts alkylation/Michael addition reaction of indoles with nitroolefin enoates catalyzed by a diphenylamine-linked bis(oxazoline)-Zn(OTf $)_{2}$ complex was investigated. This tandem reaction afforded functionalized chiral chromans in good yields with moderate to high stereoselectivities (up to 95:5 dr, up to 99\% ee).
\end{abstract}

\section{Introduction}

The development of efficient and convenient methods to access complex compounds with multiple stereogenic centers is one of the significant challenges in organic chemistry. Catalytic asymmetric tandem or cascade reactions are powerful tools to afford complex molecules with multiple stereogenic centers [1-13]. Newly developed tandem/domino reactions are increasingly applied in the synthesis of natural products and other biologically active compounds [14-16].

Dihydrocoumarins, chromans, and chromenes can be found in many natural products and synthetic molecules, and they also possess potentially useful biological properties [17-20]. The benzopyran framework has attracted considerable attention because of the importance of chromans and their biological properties. Numerous synthetic routes have been reported over the past few decades [21-32]. Chiral indolyl(nitro)chromans have been successfully synthesized in our previous study [33]. Good results were obtained in the diastereo- and enantioselective Friedel-Crafts alkylation of indoles with 3-nitro- $2 \mathrm{H}$ chromenes catalyzed by diphenylamine-linked bis(oxazoline)$\mathrm{Zn}$ (II) complexes. On the other hand, indole and its derivatives are one of the most intensively investigated classical heterocycles owing to their prevalence in bioactive compounds. Indoles have been successfully utilized in asymmetric Friedel-Crafts reactions with nitroolefin and its derivatives in previous reports [34-49]. During the preparation of this manuscript, a similar report on the enantioselective synthesis of highly substituted chromans by a Zinc(II)-catalyzed tandem Friedel-Crafts alkylation/Michael addition reaction has appeared [50]. Herein, we wish to detail our independent 
research on the asymmetric tandem Friedel-Crafts alkylation/ Michael addition reaction of indoles with nitroolefin enoates catalyzed by bis(oxazoline)-Zn(OTf $)_{2}$ complexes, resulting in functionalized chiral chromans (dihydrobenzopyrans) in moderate to high diastereoselectivities (up to 95:5 dr) and enantioselectivities (up to $99 \%$ ee).

\section{Results and Discussion}

Our initial exploratory efforts began with the optimization of the model reaction between nitroolefin enoate 1a and indole $\mathbf{2 a}$. First, a series of chiral bis(oxazoline) ligands (I-V) with $\mathrm{Zn}(\mathrm{OTf})_{2}$ as catalysts were investigated in this reaction (Figure 1). The results are summarized in Table 1. It was found that the reaction exhibited good yield and high stereoselectivity with catalysis by the $\mathbf{I}-\mathrm{Zn}(\mathrm{OTf})_{2}$ complex (Table 1, entry 1 ). Interestingly, with the ligands III-V (Table 1, entries 3-5), the reaction gave the opposite stereoselectivities probably because of the lower steric hindrance compared with I and II. Although the opposite diastereomer was obtained in good yield and stereoselectivity with the use of ligand IV, ligand I was the preferred one according to the results.

In order to increase the yield and stereoselectivity of the desired product, further screening of reaction parameters such as the ratio of substrates and temperature were investigated. When 1.5 equiv of indole was used in the reaction, a significant improvement of the yield was realized (Table 2, entry 2). The enantioselectivity of the product 3a was improved slightly by lowering the reaction temperature (Table 2, entries 7 and 8). Raising the temperature to $50{ }^{\circ} \mathrm{C}$ led to a decrease of yield, dia-

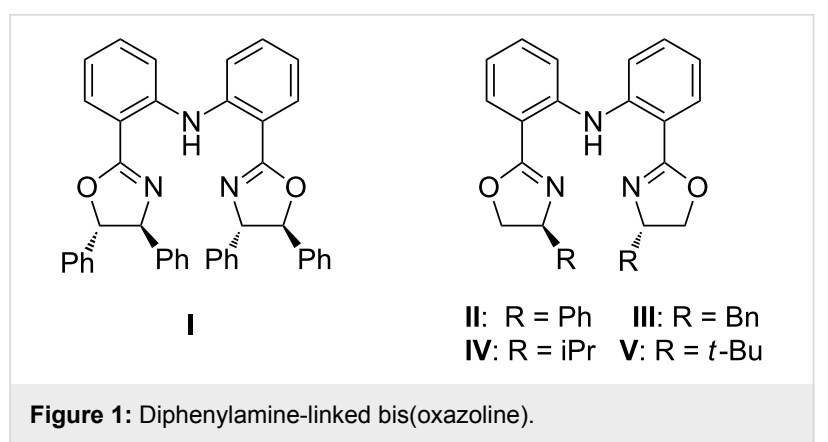

stereoselectivity and enantioselectivity. After a brief screening of the solvent, toluene was found to be the best choice.

Different additives (10 mol \%) were then tested in the presence of $10 \mathrm{~mol} \%$ of I-Zn(OTf $)_{2}$ complex. Remarkably, a substantial improvement of the yield was realized when $\mathrm{Et}_{3} \mathrm{~N}$ was added; however, the diastereo- and enantioselectivity dropped significantly (Table 3 , entry 2 ). With both $\mathrm{NH}\left(\mathrm{C}_{2} \mathrm{H}_{5}\right)_{2}$ and TMEDA, no desired product was observed (Table 3 , entries 3 and 4). DABCO led to no significant increase in yield and stereoselectivity (Table 3, entry 5). A substantial increase in enantioselectivity was observed with the use of $\mathrm{LiO} t-\mathrm{Bu}$, but the yield remained moderate (Table 3, entry 7). Among the additives probed, the best results (73\% yield, $96: 4 \mathrm{dr}$ and $89 \%$ ee) were achieved when $\mathrm{NaO} t$ - $\mathrm{Bu}$ was used as an additive in the reaction (Table 3 , entry 8).

After optimization of the reaction conditions, the substrate scope of the enantioselective Friedel-Crafts alkylation/Michael

Table 1: Effect of ligands on the asymmetric tandem Friedel-Crafts alkylation/Michael addition reaction.

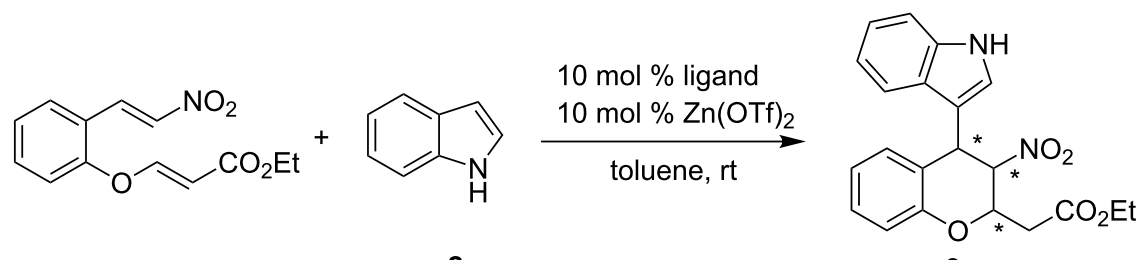

$1 \mathrm{a}$

3a

\begin{tabular}{lllll}
\hline entry $^{\mathrm{a}}$ & ligand & yield $(\%)^{\mathrm{b}}$ & $\mathrm{dr}^{\mathrm{c}}$ & ee (\%),d \\
\hline 1 & I & 48 & $96: 4$ & $83 /-$ \\
2 & II & 59 & $88: 12$ & $55 /-$ \\
3 & III & 43 & $33: 67$ & -168 \\
4 & IV & 56 & $17: 83$ & -173 \\
5 & V & 35 & $29: 71$ & $-/ 53$ \\
\hline
\end{tabular}

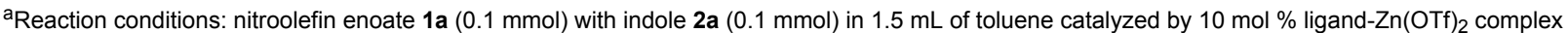
for $24 \mathrm{~h}$ at room temperature. ${ }^{b} /$ solated yields by column chromatography. ${ }^{\mathrm{C}}$ Determined by HPLC on Daicel Chiralpak IA column ( $n$-hexane/2-propanol $85: 15,0.5 \mathrm{~mL} / \mathrm{min})$. dee for the major diastereomer. 
Table 2: Optimization of reaction conditions.

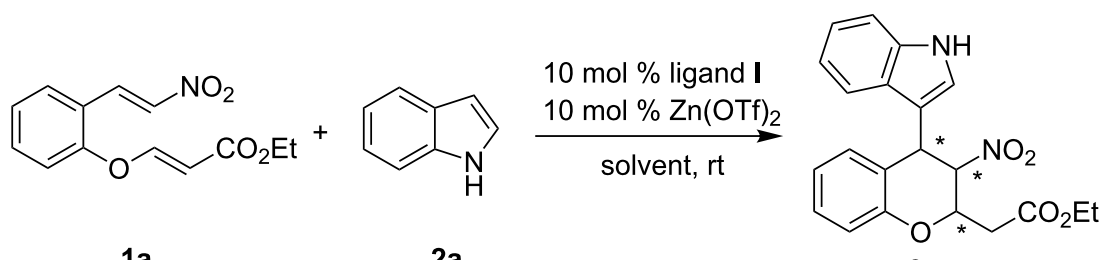

$1 \mathrm{a}$

$2 \mathbf{a}$

$3 a$

\begin{tabular}{lllll}
\hline entry $^{\mathrm{a}}$ & solvent & yield $(\%)^{\mathrm{b}}$ & $\mathrm{dr}$ & \\
\hline $1^{\mathrm{d}}$ & toluene & 48 & $96: 4$ & 83 \\
2 & toluene & 58 & $96: 4$ & 84 \\
3 & xylene & 49 & $94: 6$ & 82 \\
4 & $\alpha, \alpha, \alpha-$ trifluorotoluene & 56 & $95: 5$ & 54 \\
5 & $\mathrm{CH}_{2} \mathrm{ClCH}{ }_{2} \mathrm{Cl}$ & 37 & $89: 11$ & 69 \\
6 & THF & trace & - & - \\
$7^{\mathrm{e}}$ & toluene & 55 & $97: 3$ & 87 \\
$8^{\mathrm{f}}$ & toluene & 58 & $96: 4$ & 87 \\
$9^{g}$ & toluene & 45 & $93: 7$ & 74 \\
\hline
\end{tabular}

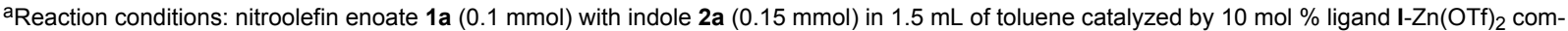
plex for $24 \mathrm{~h}$ at room temperature. ${ }^{\mathrm{b}}$ Isolated yields by column chromatography. 'Determined by HPLC on Daicel Chiralpak IA column ( $n$-hexane/

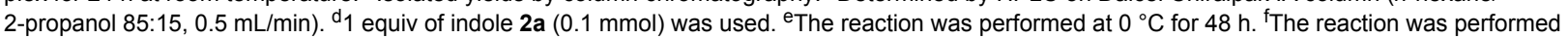
at $-10{ }^{\circ} \mathrm{C}$ for $48 \mathrm{~h}$. 9The reaction was performed at $50{ }^{\circ} \mathrm{C}$ for $24 \mathrm{~h}$.

Table 3: Effect of additives on asymmetric tandem Friedel-Crafts alkylation/Michael addition reaction.

\begin{tabular}{|c|c|c|c|c|}
\hline & $1 a$ & $\begin{array}{r}\mathrm{H} \\
\mathbf{2 a}\end{array}$ & $\begin{array}{l}\text { d I } \\
\underset{\text { ivf })_{2}}{\stackrel{\text { ive }}{\longrightarrow}}\end{array}$ & \\
\hline entry $^{a}$ & additive & yield $(\%)^{b}$ & $d r^{c}$ & ee $(\%)^{c}$ \\
\hline 1 & - & 58 & $96: 4$ & 83 \\
\hline 2 & $\mathrm{Et}_{3} \mathrm{~N}$ & 76 & $79: 21$ & 63 \\
\hline 3 & $\mathrm{NH}\left(\mathrm{C}_{2} \mathrm{H}_{5}\right)_{2}$ & 0 & - & - \\
\hline 4 & TMEDA & 0 & - & - \\
\hline 5 & DABCO & 41 & $93: 7$ & 82 \\
\hline 6 & $\mathrm{CsCO}_{3}$ & 62 & $98: 2$ & 80 \\
\hline 7 & $\mathrm{LiO} t-\mathrm{Bu}$ & 47 & $95: 5$ & 92 \\
\hline 8 & $\mathrm{NaO} t-\mathrm{Bu}$ & 73 & $96: 4$ & 89 \\
\hline 9 & $\mathrm{KOt}-\mathrm{Bu}$ & 62 & $96: 4$ & 84 \\
\hline
\end{tabular}

aReaction conditions: nitroolefin enoate $1 \mathrm{a}(0.1 \mathrm{mmol})$ with indole $2 \mathrm{a}(0.15 \mathrm{mmol})$ in $1.5 \mathrm{~mL}$ of toluene catalyzed by $10 \mathrm{~mol} \%$ ligand $\mathrm{I}-\mathrm{Zn}(\mathrm{OTf})_{2} \mathrm{Com}-$ plex with $10 \mathrm{~mol} \%$ additive for $24 \mathrm{~h}$ at room temperature. ${ }^{\mathrm{b}}$ Isolated yields by column chromatography. ${ }^{\mathrm{C}}$ Determined by HPLC on Daicel Chiralpak IA column ( $n$-hexane/2-propanol 85:15, $0.5 \mathrm{~mL} / \mathrm{min})$.

addition of nitroolefin enoates 1 with indoles $\mathbf{2}$ was explored. The results are summarized in Table 4 (see Supporting Information File 1 for full experimental data). Both electron withdrawing and electron-rich substituents in the 5-position of the indole caused moderate decrease in enantioselectivity and diastereoselectivity (Table 4, entries $2-4$ ). Nitroolefin enoates $\mathbf{1 b}$ or 1c with chlorine or bromine on the aromatic ring reacted smoothly to afford products $\mathbf{3 f}$ or $\mathbf{3 g}$ with good yields and ste- 
Table 4: Asymmetric tandem Friedel-Crafts alkylation/Michael addition reaction of nitroolefin enoates with indoles.<smiles>[R]c1cc([R])c(O/C=C/C(=O)OCC)c(/C=C/[N+](=O)[O-])c1</smiles>

1

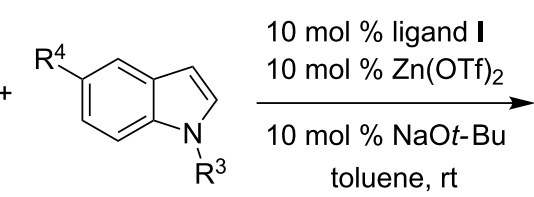

2<smiles></smiles>

3

\begin{tabular}{|c|c|c|c|c|c|c|c|c|}
\hline entry $^{a}$ & $\mathrm{R}^{1}$ & $R^{2}$ & $\mathrm{R}^{3}$ & $\mathrm{R}^{4}$ & product & yield $(\%)^{b}$ & $\mathrm{dr}^{\mathrm{C}}$ & ee $(\%)^{c, d}$ \\
\hline $1^{e}$ & $\mathrm{H}$ & $\mathrm{H}$ & $\mathrm{H}$ & $\mathrm{H}$ & $3 a$ & 73 & $96: 4$ & 89 \\
\hline 2 & $\mathrm{H}$ & $\mathrm{H}$ & $\mathrm{H}$ & $\mathrm{CH}_{3}$ & $3 b$ & 69 & $90: 10$ & 80 \\
\hline 3 & $\mathrm{H}$ & $\mathrm{H}$ & $\mathrm{H}$ & $\mathrm{OCH}_{3}$ & $3 c$ & 76 & $93: 7^{f}$ & 72 \\
\hline 4 & $\mathrm{H}$ & $\mathrm{H}$ & $\mathrm{H}$ & $\mathrm{Cl}$ & $3 d$ & 49 & $82: 18^{f}$ & 62 \\
\hline 5 & $\mathrm{H}$ & $\mathrm{H}$ & $\mathrm{CH}_{3}$ & $\mathrm{H}$ & $3 e$ & 60 & $88: 12^{f}$ & 67 \\
\hline 6 & $\mathrm{H}$ & $\mathrm{Cl}$ & $\mathrm{H}$ & $\mathrm{H}$ & $3 f$ & 65 & $95: 5$ & 91 \\
\hline 7 & $\mathrm{H}$ & $\mathrm{Br}$ & $\mathrm{H}$ & $\mathrm{H}$ & $3 g$ & 66 & $93: 7$ & 87 \\
\hline 8 & $\mathrm{H}$ & $\mathrm{NO}_{2}$ & $\mathrm{H}$ & $\mathrm{H}$ & $3 h$ & 56 & $85: 15^{f}$ & 80 \\
\hline 99 & $\mathrm{OCH}_{3}$ & $\mathrm{H}$ & $\mathrm{H}$ & $\mathrm{H}$ & $3 \mathbf{i}$ & 12 & $-^{\mathrm{h}}$ & 24 \\
\hline $10^{9}$ & $\mathrm{OC}_{2} \mathrm{H}_{5}$ & $\mathrm{H}$ & $\mathrm{H}$ & $\mathrm{H}$ & $3 \mathbf{j}$ & 47 & $85: 15^{f}$ & 31 \\
\hline 11 & $\mathrm{Br}$ & $\mathrm{Br}$ & $\mathrm{H}$ & $\mathrm{H}$ & $3 k$ & 21 & $72: 28$ & 57 \\
\hline
\end{tabular}

aReaction conditions: nitroolefin enoates $1(0.2 \mathrm{mmol})$ with indoles $2(0.3 \mathrm{mmol})$ in $3 \mathrm{~mL}$ of toluene catalyzed by $10 \mathrm{~mol} \%$ ligand-Zn(OTf $)_{2}$ complex with $10 \mathrm{~mol} \% \mathrm{NaOt}$-Bu for $24 \mathrm{~h}$ at room temperature. ${ }^{\mathrm{b}}$ lsolated yields by column chromatography. ${ }^{\mathrm{C}}$ Determined by HPLC. ${ }^{\mathrm{d}}$ ee for the major diastereomer. ${ }^{e}$ Nitroolefin enoate $1 \mathrm{a}(0.1 \mathrm{mmol})$ with indole $2 \mathrm{a}(0.15 \mathrm{mmol}){ }^{\mathrm{f}}$ Determined by the weight ratio of isolated diastereomers. ${ }^{9}$ The reaction time was $96 \mathrm{~h}$. ${ }^{\mathrm{T}}$ The minor diastereomer was not detected.

reoselectivities (Table 4, entries 6 and 7). We found that incorporation of an electron-donating methoxy group on the aromatic ring of the nitroolefin enaote had a significant effect on both yield and stereoselectivity. In the case of methoxysubstituted nitroolefin enoates 1e (Table 4, entry 9), the yield of the product $3 \mathbf{i}$ decreased to $12 \%$ and the enantioselectivity decreased to $24 \%$ even though the reaction time was prolonged to $96 \mathrm{~h}$. A similar result was observed in the case of product $\mathbf{3 j}$ (Table 4, entry 10). A steric effect was also observed in this reaction. When the substrate $1 \mathrm{~g}$ bearing two sterically hindered bromine atoms on the phenyl ring was used, the yield and stereoselectivity of the desired product $\mathbf{3 k}$ decreased significantly (Table 4, entry 11). The configuration of the major diastereomer of $\mathbf{3 g}$ was determined to be $\mathrm{C} 15(S), \mathrm{C} 16(R), \mathrm{C} 17(S)$ (Figure 2), and those of other products were assigned by analogy [51].

The results of the substrate scope are unsatisfactory. The yields of the desired products 3 were affected by the side products 4 , which were the Friedel-Crafts alkylation products of nitroolefin enoates and indoles. Fortunately, it was found that the model Friedel-Crafts alkylation product $\mathbf{4 a}$, which was isolated, could be transformed to the desired cycloadduct 3a with high stereoselectivity in the presence of 5 equiv of $\mathrm{Et}_{3} \mathrm{~N}$ at room tempera-

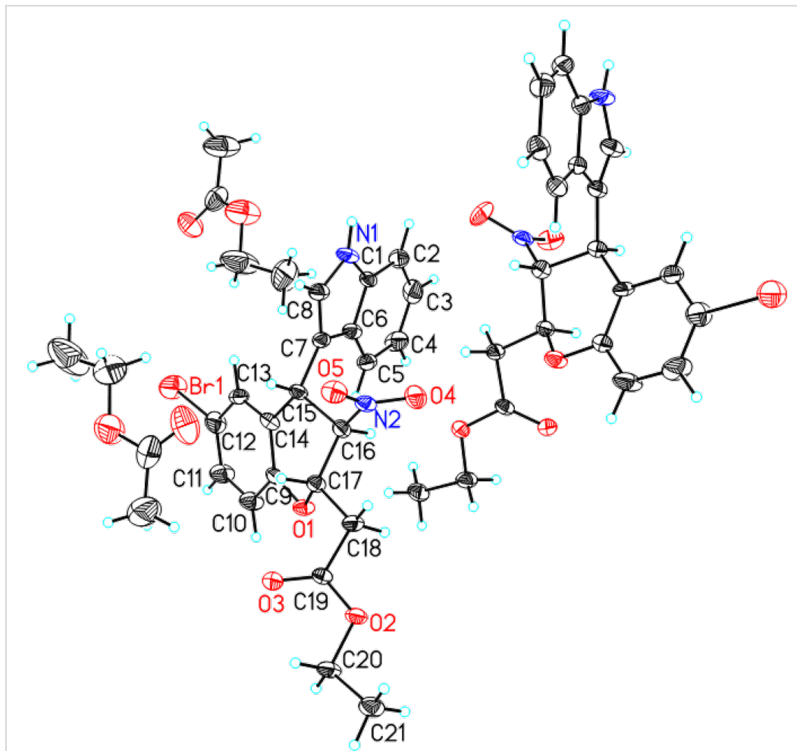

Figure 2: X-ray crystal structure of the major diastereomer of $\mathbf{3 g}$ (one symmetric molecule and two solvent molecules are not labeled for clarity).

ture (Scheme 1). With the success of this model reaction, the substrate scope in Table 4 was reinvestigated. The corresponding reactions proceeded smoothly to afford desired prod- 
ucts 3 and side products 4 at $-10{ }^{\circ} \mathrm{C}$. After the nitroolefin enoates were consumed, 5 equiv of $\mathrm{Et}_{3} \mathrm{~N}$ was added to the reaction at room temperature. The new results are summarized in Table 5. All reactions proceeded smoothly affording desired products 3 with good to excellent yields. However, the diastereoselectivities of the products 3 decreased in all cases. Excellent enantioselectivities were observed with indoles bearing electron-rich substituents (Table 5, entries 2 and 3). Both the electron-withdrawing and electron-rich substituents on the aromatic ring of nitroolefin enoates afforded $\mathbf{3}$ in low diastereoselectivities (Table 5, entries 6-10). Good to excellent enantioselectivities were observed in these cases. $\mathbf{3 k}$ was obtained in moderate stereoselectivity (Table 5, entry 11).<smiles>CCN(C)CCOc1ccccc1[C@H](C[N+](=O)[O-])c1c[nH]c2ccccc12</smiles>

4a

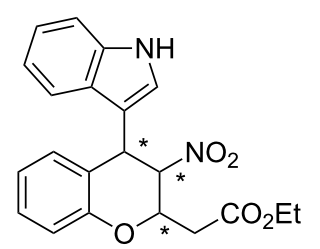

3a
Scheme 1: The transformation of Friedel-Crafts alkylation product 4a to cycloadduct $\mathbf{3 a}$.

\section{Conclusion}

In conclusion, we have developed a convenient catalytic asymmetric tandem Friedel-Crafts alkylation/Michael addition reaction of nitroolefin enoates 1 with indoles $\mathbf{2}$ catalyzed by a tridentate bis(oxazoline) I-Zn(OTf) 2 complex. Moderate to high stereoselectivities (up to $95: 5 \mathrm{dr}$, up to $99 \%$ ee) and good to excellent yields of the functionalized chiral chromans were obtained. Further applications of these catalysts in other reactions are underway in our laboratory.

\section{Experimental}

General procedure A for the catalytic asymmetric tandem Friedel-Crafts alkylation/Michael addition reaction of indoles with nitroolefin enoates: Into a dried Schlenk tube were added $\mathrm{Zn}(\mathrm{OTf})_{2}$ (7.3 mg, $\left.0.02 \mathrm{mmol}\right)$, ligand I (12.2 mg, $\left.0.02 \mathrm{mmol}\right)$ and $\mathrm{NaO} t$-Bu $(1.9 \mathrm{mg}, 0.02 \mathrm{mmol})$ under argon followed by the addition of toluene $(3 \mathrm{~mL})$. The solution was stirred at room temperature for $0.5 \mathrm{~h}$, and then nitroolefin enoate $1(0.2 \mathrm{mmol})$ was added. The mixture was stirred for $10 \mathrm{~min}$ then the indole 2 $(0.3 \mathrm{mmol})$ was added. After stirring for $48 \mathrm{~h}$ at room temperature, the solvent was removed under vacuum. Purification by column chromatography afforded the desired products 3 .

General procedure B for the catalytic asymmetric tandem Friedel-Crafts alkylation/Michael addition reaction of indoles

Table 5: Asymmetric tandem Friedel-Crafts alkylation/Michael addition reaction of nitroolefin enoates with indoles.<smiles>[R]c1cc([R])c(O/C=C/C(=O)OCC)c(/C=C/[N+](=O)[O-])c1</smiles>

\begin{tabular}{lllllllll}
\hline entry & $\mathrm{R}^{\mathbf{a}}$ & $\mathrm{R}^{2}$ & $\mathrm{R}^{3}$ & $\mathrm{R}^{4}$ & product & Yield $(\%)^{\mathrm{b}}$ & $\mathrm{drc}^{\mathrm{c}}$ & ee $(\%)^{\mathrm{c}, \mathrm{d}}$ \\
\hline 1 & $\mathrm{H}$ & $\mathrm{H}$ & $\mathrm{H}$ & $\mathrm{H}$ & $\mathbf{3 a}$ & 88 & $92: 8$ & $92 / 88$ \\
2 & $\mathrm{H}$ & $\mathrm{H}$ & $\mathrm{H}$ & $\mathrm{CH}_{3}$ & $\mathbf{3 b}$ & 88 & $44: 56^{\mathrm{e}}$ & $62 / 98$ \\
3 & $\mathrm{H}$ & $\mathrm{H}$ & $\mathrm{H}$ & $\mathrm{OCH}_{3}$ & $\mathbf{3 c}$ & 96 & $28: 72^{\mathrm{e}}$ & $73 / 99$ \\
4 & $\mathrm{H}$ & $\mathrm{H}$ & $\mathrm{H}$ & $\mathrm{Cl}$ & $\mathbf{3 d}$ & 58 & $76: 24$ & $82 / 53$ \\
5 & $\mathrm{H}$ & $\mathrm{H}$ & $\mathrm{CH}_{3}$ & $\mathrm{H}$ & $\mathbf{3 e}$ & 89 & $82: 18^{\mathrm{e}}$ & $65 / 79$ \\
6 & $\mathrm{H}$ & $\mathrm{Cl}$ & $\mathrm{H}$ & $\mathrm{H}$ & $\mathbf{3 f}$ & 86 & $74: 26$ & $95 / 95$ \\
7 & $\mathrm{H}$ & $\mathrm{Br}$ & $\mathrm{H}$ & $\mathrm{H}$ & $\mathbf{3 g}$ & 100 & $60: 40$ & $91 / 93$ \\
8 & $\mathrm{H}$ & $\mathrm{NO}$ & $\mathrm{H}$ & $\mathrm{H}$ & $\mathbf{3 h}$ & 89 & $49: 51$ & $95 / 95$ \\
9 & $\mathrm{OCH}_{3}$ & $\mathrm{H}$ & $\mathrm{H}$ & $\mathrm{H}$ & $\mathbf{3 i}$ & 85 & $58: 42^{\mathrm{e}}$ & $39 / 83$ \\
10 & $\mathrm{OC}_{2} \mathrm{H}_{5}$ & $\mathrm{H}$ & $\mathrm{H}$ & $\mathrm{H}$ & $\mathbf{3 j}$ & 94 & $54: 46^{\mathrm{e}}$ & $30 / 97$ \\
11 & $\mathrm{Br}$ & $\mathrm{Br}$ & $\mathrm{H}$ & $\mathrm{H}$ & $\mathbf{3 k}$ & 75 & $38: 62$ & $53 / 90$ \\
\hline
\end{tabular}

aReaction conditions: nitroolefin enoate $1 \mathbf{a}(0.2 \mathrm{mmol})$ with indole $2 \mathrm{a}(0.3 \mathrm{mmol})$ in $3 \mathrm{~mL}$ of toluene catalyzed by $10 \mathrm{~mol} \%$ ligand-Zn(OTf) 2 complex for $72 \mathrm{~h}$ at $-10^{\circ} \mathrm{C}$. Subsequently, 5 equiv of $\mathrm{Et}_{3} \mathrm{~N}$ was added. blsolated yields by column chromatography. ${ }^{\mathrm{C}}$ Determined by HPLC. ${ }^{\mathrm{d}}$ ee for both diastereomers. ${ }^{e}$ Determined by the weight ratio of isolated diastereomers. 
with nitroolefin enoates: Into a dried Schlenk tube were added $\mathrm{Zn}(\mathrm{OTf})_{2}(7.3 \mathrm{mg}, 0.02 \mathrm{mmol})$ and ligand I (12.2 mg, $0.02 \mathrm{mmol}$ ) under argon followed by the addition of toluene $(3 \mathrm{~mL})$. The solution was stirred at room temperature for $0.5 \mathrm{~h}$ and then nitroolefin enoate $\mathbf{1}(0.2 \mathrm{mmol})$ was added. The mixture was stirred for $10 \mathrm{~min}$ then the indole $2(0.3 \mathrm{mmol})$ was added. After stirring for $72 \mathrm{~h}$ at $-10{ }^{\circ} \mathrm{C}, \mathrm{Et}_{3} \mathrm{~N}$ (100 mg, $\left.1 \mathrm{mmol}\right)$ was added, and the mixture was stirred for another $24 \mathrm{~h}$ at room temperature. The solvent was removed under vacuum. Purification by column chromatography afforded the desired products 3 .

\section{Supporting Information}

\section{Supporting Information File 1}

Characterization data, copies of NMR spectra and HPLC

chromatographs of products $\mathbf{3}$.

[http://www.beilstein-journals.org/bjoc/content/

supplementary/1860-5397-9-137-S1.pdf]

\section{Supporting Information File 2}

Crystallographic data of compound $\mathbf{3 g}$.

[http://www.beilstein-journals.org/bjoc/content/

supplementary/1860-5397-9-137-S2.cif]

\section{Acknowledgements}

We are grateful for financial support from the National Natural Science Foundation of China (Grant Nos. 20772006, 21072020), the Science and Technology Innovation Program of Beijing Institute of Technology (Grant No. 2011CX01008) and the Development Program for Distinguished Young and Middle-aged Teachers of Beijing Institute of Technology.

\section{References}

1. Posner, G. H. Chem. Rev. 1986, 86, 831-844. doi:10.1021/cr00075a007

2. Tietze, L. F.; Beifuss, U. Angew. Chem., Int. Ed. Engl. 1993, 32, 131-163. doi:10.1002/anie.199301313

3. Tietze, L. F. Chem. Rev. 1996, 96, 115-136. doi:10.1021/cr950027e

4. Wasilke, J.-C.; Obrey, S. J.; Baker, R. T.; Bazan, G. C. Chem. Rev. 2005, 105, 1001-1020. doi:10.1021/cr020018n

5. Ramón, D. J.; Yus, M. Angew. Chem., Int. Ed. 2005, 44, 1602-1634. doi:10.1002/anie.200460548

6. Pellissier, H. Tetrahedron 2006, 62, 1619-1665. doi:10.1016/j.tet.2005.10.040

7. Pellissier, H. Tetrahedron 2006, 62, 2143-2173. doi:10.1016/j.tet.2005.10.041

8. Guo, H.-C.; Ma, J.-A. Angew. Chem., Int. Ed. 2006, 45, 354-366. doi:10.1002/anie.200500195

9. Enders, D.; Grondal, C.; Hüttl, M. R. M. Angew. Chem., Int. Ed. 2007, 46, 1570-1581. doi:10.1002/anie.200603129
10. Grondal, C.; Jeanty, M.; Enders, D. Nat. Chem. 2010, 2, 167-178. doi:10.1038/nchem.539

11. Moyano, A.; Rios, R. Chem. Rev. 2011, 111, 4703-4832. doi:10.1021/cr100348t

12. Pellissier, H. Adv. Synth. Catal. 2012, 354, 237-294. doi:10.1002/adsc.201100714

13. Schreiber, S. L. Science 2000, 287, 1964-1969. doi:10.1126/science.287.5460.1964

14. Nicolaou, K. C.; Edmonds, D. J.; Bulger, P. G. Angew. Chem., Int. Ed. 2006, 45, 7134-7186. doi:10.1002/anie.200601872

15. de Figueiredo, R. M.; Christmann, M. Eur. J. Org. Chem. 2007, 2575-2600. doi:10.1002/ejoc.200700032

16. Rueping, M.; Dufour, J.; Schoepke, F. R. Green Chem. 2011, 13, 1084-1105. doi:10.1039/c1gc15027h

17. Conti, C.; Monaco, L. P.; Desideri, N. Bioorg. Med. Chem. 2011, 19, 7357-7364. doi:10.1016/j.bmc.2011.10.060

18. Schweizer, E. E.; Meeder-Nycz, O. In Chromenes, Chromanes, Chromones; Ellis, G. P., Ed.; Wiley-Interscience: New York, NY, 1977; pp 11-139.

19. Engler, T. A.; LaTessa, K. O.; Iyengar, R.; Chai, W.; Agrios, K. Bioorg. Med. Chem. 1996, 4, 1755-1769. doi:10.1016/0968-0896(96)00192-7

20. Broggini, G.; Folcio, F.; Sardone, N.; Sonzogni, M.; Zecchi, G. Tetrahedron: Asymmetry 1996, 7, 797-806. doi:10.1016/0957-4166(96)00076-6

21. Sugimoto, H.; Nakamura, S.; Ohwada, T. Adv. Synth. Catal. 2007, 349, 669-679. doi:10.1002/adsc.200600508

22. Fukamizu, K.; Miyake, Y.; Nishibayashi, Y. J. Am. Chem. Soc. 2008, 130, 10498-10499. doi:10.1021/ja8038745

23. Xu, D.-Q.; Wang, Y.-F.; Luo, S.-P.; Zhang, S.; Zhong, A.-G.; Chen, H.; Xu, Z.-Y. Adv. Synth. Catal. 2008, 350, 2610-2616. doi:10.1002/adsc. 200800535

24. Ramachary, D. B.; Sakthidevi, R. Chem.-Eur. J. 2009, 15, 4516-4522. doi:10.1002/chem.200900066

25. Xie, J.-W.; Huang, X.; Fan, L.-P.; Xu, D.-C.; Li, X.-S.; Su, H.; Wen, Y.-H. Adv. Synth. Catal. 2009, 351, 3077-3082. doi:10.1002/adsc.200900579

26. Shen, H. C. Tetrahedron 2009, 65, 3931-3952. doi:10.1016/j.tet.2009.02.002

27. Ferreira, S. B.; da Silva, F. C.; Pinto, A. C.; Gonzaga, D. T. G.; Ferreira, V. F. J. Heterocycl. Chem. 2009, 46, 1080-1097. doi:10.1002/jhet.232

28. Lim, H. J.; RajanBabu, T. V. Org. Lett. 2009, 11, 2924-2927. doi:10.1021/ol900961m

29. Pearson, E. L.; Kanizaj, N.; Willis, A. C.; Paddon-Row, M. N.; Sherburn, M. S. Chem.-Eur. J. 2010, 16, 8280-8284. doi:10.1002/chem.201001176

30. Rueping, M.; Lin, M.-Y. Chem.-Eur. J. 2010, 16, 4169-4172. doi:10.1002/chem.201000203

31. Zhang, X.; Zhang, S.; Wang, W. Angew. Chem., Int. Ed. 2010, 49, 1481-1484. doi:10.1002/anie.200906050

32. Lu, L.-Q.; Chen, J.-R.; Xiao, W.-J. Acc. Chem. Res. 2012, 45 , 1278-1293. doi:10.1021/ar200338s

33. Jia, Y.; Yang, W.; Du, D.-M. Org. Biomol. Chem. 2012, 10, 4739-4746. doi:10.1039/c2ob25360g

34. Poulsen, T. B.; Jørgensen, K. A. Chem. Rev. 2008, 108, 2903-2915. doi:10.1021/cr078372e

35. Bandini, M.; Eichholzer, A. Angew. Chem., Int. Ed. 2009, 48, 9608-9644. doi:10.1002/anie.200901843 
36. You, S.-L.; Cai, Q.; Zeng, M. Chem. Soc. Rev. 2009, 38, 2190-2201. doi:10.1039/b817310a

37. Herrera, R. P.; Sgarzani, V.; Bernardi, L.; Ricci, A.

Angew. Chem., Int. Ed. 2005, 44, 6576-6579.

doi:10.1002/anie.200500227

38. Jia, Y.-X.; Zhu, S.-F.; Yang, Y.; Zhou, Q.-L. J. Org. Chem. 2006, 71, 75-80. doi:10.1021/jo0516537

39. Lu, S.-F.; Du, D.-M.; Xu, J. Org. Lett. 2006, 8, 2115-2118. doi:10.1021/ol060586f

40. Singh, P. K.; Bisai, A.; Singh, V. K. Tetrahedron Lett. 2007, 48, 1127-1129. doi:10.1016/j.tetlet.2006.12.081

41. Liu, H.; Lu, S.-F.; Xu, J.; Du, D.-M. Chem.-Asian J. 2008, 3, 1111-1121. doi:10.1002/asia.200800071

42. Ganesh, M.; Seidel, D. J. Am. Chem. Soc. 2008, 130, 16464-16465. doi:10.1021/ja8063292

43. Itoh, J.; Fuchibe, K.; Akiyama, T. Angew. Chem., Int. Ed. 2008, 47, 4016-4018. doi:10.1002/anie.200800770

44. McKeon, S. C.; Müller-Bunz, H.; Guiry, P. J. Eur. J. Org. Chem. 2009, 4833-4841. doi:10.1002/ejoc.200900683

45. Yokoyama, N.; Arai, T. Chem. Commun. 2009, 3285-3287. doi:10.1039/b904275j

46. Liu, H.; Du, D.-M. Adv. Synth. Catal. 2010, 352, 1113-1118. doi:10.1002/adsc.201000111

47. Liu, H.; Du, D.-M. Eur. J. Org. Chem. 2010, 2121-2131. doi:10.1002/ejoc.200901434

48. Guo, F.; Lai, G.; Xiong, S.; Wang, S.; Wang, Z. Chem.-Eur. J. 2010, 16, 6438-6441. doi:10.1002/chem.201000540

49. Peng, J.; Du, D.-M. Eur. J. Org. Chem. 2012, 4042-4051. doi:10.1002/ejoc.201200382

50. Li, C.; Liu, F.-L.; Zou, Y.-Q.; Lu, L.-Q.; Chen, J.-R.; Xiao, W.-J. Synthesis 2013, 45, 601-608. doi:10.1055/s-0032-1318200

51. CCDC-936409 contains the supplementary crystallographic data for this paper. These data can be obtained free of charge from The Cambridge Crystallographic DataCentre via http://www.ccdc.cam.ac.uk/ data_request/cif.

\section{License and Terms}

This is an Open Access article under the terms of the Creative Commons Attribution License

(http://creativecommons.org/licenses/by/2.0), which permits unrestricted use, distribution, and reproduction in any medium, provided the original work is properly cited.

The license is subject to the Beilstein Journal of Organic Chemistry terms and conditions:

(http://www.beilstein-journals.org/bjoc)

The definitive version of this article is the electronic one which can be found at: $\underline{\text { doi:10.3762/bjoc. } 9.137}$ 